\title{
Isolated perforation of fourth part of Duodenum following Blunt Abdominal Trauma
}

\author{
Maharjan $\mathrm{P}^{1}$, Bharti SV${ }^{1}$, Khadka $\mathrm{DB}^{1}$, Karki $\mathrm{A}^{1}$, Gnyawali $\mathrm{A}^{1}$
}

\section{ABSTRACT}

Introduction: Isolated duodenal injuries are rare in blunt abdominal trauma. These present a significant challenge for management because of the associated injuries and its difficult anatomical accessibility. Case presentation: A 20years male presented to the Emergency department following a bike accident sustaining injury over face, chest and abdomen, 6hours after the incident. His vitals were unstable so he was resuscitated and admitted in Intensive Care Unit. He had generalized abdominal tenderness without rigidity. Contrast enhanced computed tomography of abdomen and pelvis was suggestive of hollow viscus perforation. He underwent exploratory laparotomy and primary repair for isolated perforation at fourth part of duodenum. He was discharged on his ninth postoperative day. Conclusion: Rare injuries following blunt abdominal trauma should be considered and early intervention is necessary.

Keywords: Blunt Abdominal Trauma, Duodenal Perforation, Primary Repair

Authors:

1. Dr. Prabir Maharjan

2. Dr. Shiv Vansh Bharti

3. Dr. Digbijay Bikram Khadka

4. Dr. Anup Karki

5. Dr. Arun Gnyawali

${ }^{1}$ Department of Surgery, Nepalgunj Medical College and Teaching Hospital, Kohalpur, Banke

\section{Address for Correspondence:}

Dr. Prabir Maharjan

Department of Surgery

Nepalgunj Medical College and Teaching Hospital

Kohalpur, Banke

E-mail: prabirstx493@gmail.com

\section{INTRODUCTION}

Blunt abdominal trauma is one of the common presentations of patients in the Emergency Department of any institution. It is common in young age group and most injuries occur following road traffic accidents. Spleen and Liver are the common organs injured following such injury. ${ }^{1}$ However, injury to small intestine is rare and isolated injury to the duodenum represents only 2-20\%. Concomitant intra-abdominal injuries to pancreas and liver are usually associated with duodenal injuries. The retroperitoneal location of the duodenum protects the duodenum from external injury and comprise $3.7 \%$ of patients undergoing laparotomy for blunt or penetrating trauma. Blunt duodenal injury is associated with significant mortality (6$25 \%$ ) and morbidity (30-60\%) due to its delayed diagnosis. Preoperative assessment with computed tomography scan in stable patients may not accurately delineate the particular injuries always given that retroperitoneal hematoma makes the interpretation more challenging. Surgical repair can range from simple repair, Roux-en-Y duodeno-jejunostomies, T-tube decompression and pancreatico-duodenectomy. ${ }^{2}$

\section{CASE REPORT}

A 20years male presented to the Emergency department of
Nepalgunj Medical College and Teaching Hospital, following a bike accident sustaining injury over the face, chest and abdomen, 6 hours after the incident. He was examined using the ATLS protocol. The patient had no airway and breathing issues. However, his blood pressure was $80 / 50 \mathrm{mmHg}$ and pulse was 110 beats per minute. Considering his unstable hemodynamic status, he was resuscitated and admitted in Intensive Care Unit. There was no history of head injury, bleeding from any part of the body nor loss of consciousness. He had no pallor. He had bruises over his left hypochondrium. He had generalized abdominal tenderness without rigidity. Digital rectal examination and other systemic examinations were unremarkable. Eventually, the patient underwent Contrast enhanced computed tomography of abdomen and pelvis after resuscitation. The reports showed gross intraperitoneal low attenuating collection with few locules of free air within suggestive of Hollow Viscus Perforation. He underwent emergency exploratory laparotomy. Gross intraperitoneal collection with bile mixed hemorrhagic fluid approximately 2.5 liters was observed. Perforation approximately $3 \times 3 \mathrm{~cm}^{2}$ over the anterior wall of the fourth part of duodenum was found and repaired. A feeding jejunostomy was placed. There were no intraoperative issues. His post-operative period was uneventful. 

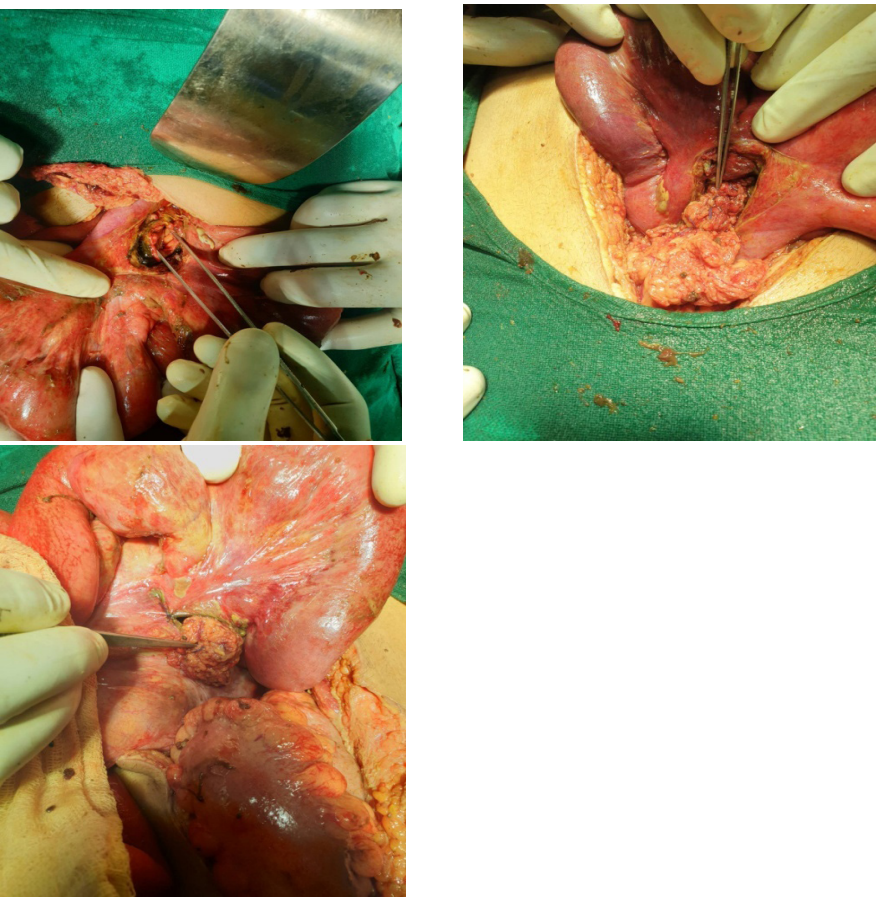

\section{DISCUSSION}

Duodenal perforation is a potentially life-threatening condition. Due to its relatively well protected anatomical site, duodenum is less commonly injured compared to jejunum after a blunt trauma. A six year study by Ballard RB et al (1997) was conducted to assess blunt duodenal rupture in 103,864 patients entered into a statewide trauma registry in which the author identified an incidence of $0.2 \%$ (206 patients). Second part of duodenum was the most commonly injured segment having $27 \%$ incidence while third part of duodenum with $17 \%$ incidence. Similarly, injury to fourth part of duodenum was reported $13 \%$. All these duodenal injuries were associated with other injuries. Isolated injury to the duodenum was observed to be only $1.45 \% .^{3-5}$

The risk of associated injuries to the main pancreatic duct, common bile duct, portal vein, abdominal aorta, inferior venacava and superior mesenteric vessels, as well as increased risk of spleen or diaphragmatic rupture makes duodenal injury potentially which were not evident in our patient. Theoretically, duodenal perforation should be associated with amylase or other digestive enzyme leakage but due to its low specificity, serial assessment of the serum amylase would be time consuming and inconclusive. Our patient had a serum amylase level of $130.5 \mathrm{U} / \mathrm{L}$. An abdominal CECT scan with oral and intravenous contrast is ideal for blunt trauma injuries. It can be used to accurately diagnose retroperitoneal injuries and is sensitive to small amounts of retroperitoneal air, blood or leaked contrast from the injured duodenum. ${ }^{2}$ Our patient also underwent CECT abdomen and pelvis which identified locules of free air which assisted us to proceed with the decision of surgery.

Duodenal injuries can range from intramural hematoma to complete transection and devascularization of the duodenum and are graded I to $\mathrm{V}$ by the American Association for the Surgery of Trauma. Our patient had a grade II duodenal injury with a laceration of $<50 \%$ of the circumference. ${ }^{6}$ (Table I)

\begin{tabular}{|c|c|c|}
\hline Grade & & Injury Description \\
\hline \multirow{2}{*}{1} & Hematoma & Involving single portion of duodenum \\
\hline & laceration & Partial thickness, no perforation \\
\hline \multirow{2}{*}{ ॥ } & Hematoma & Involving more than one portion \\
\hline & Laceration & Disruption of $<50 \%$ of circumference \\
\hline \multirow[b]{2}{*}{ III } & & Disruption $50-75 \%$ of circumference of D2 \\
\hline & Laceration & $\begin{array}{l}\text { Disruption of } 50-100 \% \text { of circumference of D1, D3 } \\
\text { and D4 }\end{array}$ \\
\hline \multirow{2}{*}{ IV } & Laceration & \\
\hline & & Involving ampulla or distal common bile duct \\
\hline & Laceration & Massive disruption of duodenopancreatic complex \\
\hline & Vascular & Devascularization of duodenum \\
\hline
\end{tabular}

Table I: Grading of Duodenal Injury (AAST) ${ }^{2}$

Ansari D et al (2019) has written an excellent narrative review on the diagnosis and management of duodenal perforations. The review focuses more on the multiple etiologies associated with duodenal perforations and states traumatic duodenal perforations incidence less than $2 \%$ of all abdominal injuries, majority being secondary to penetrating injury. The author also advised various management strategies as either conservative, endoscopic or surgical on the basis of the size of the perforation and hemodynamic status of the patient. However, the review doesn't emphasize on the management according to the part of the duodenum involved. It also suggested to either attempt primary closure, pedicled omental flap (Cellan-Jones repair) or free omental plug (Graham patch) for surgical repair of the perforation. Our patient underwent classical Graham Patch repair with no significant postoperative complications. ${ }^{7}$ Omondi $\mathrm{M}$ et al (2020) reported a case on a 22 years old male who presented to the hospital in a very similar way as our patient and during the laparotomy, a perforation at fourth part of duodenum on the anterior wall was found with no associated injuries. The lacerated edges were freshened and repaired in a single layer using interrupted 3-0 polyglactin suture and the patient had a decent postoperative period. ${ }^{3}$ Celik A et al (2010) published case series and literature review on the management of isolated duodenal rupture due to blunt abdominal trauma in which he discussed about three cases with grade III to grade IV injuries in the third part of duodenum. One of the patients underwent HeinekeMikulicz pyloroplasty like primary repair, second and the third patients underwent primary repair and pyloric exclusion with gastrojejunostomy. ${ }^{2}$ Huang $\mathrm{CL}$ et al (2011) reported a case of a 12 years old boy struck with the handlebar of a bicycle who underwent CECT abdomen which revealed thickening of fourth part of duodenum and free air and fluid collection in the retroperitoneum. This patient was operated laparoscopically 
using five ports. The surgeon visualized $50 \%$ circumferential laceration over the fourth part of duodenum. Primary closure was performed with interrupted 3-o polydioxanone. ${ }^{8}$ As per most reports in the literature, patients with blunt duodenal hematoma are initially managed non operatively, whereas immediate surgical intervention is necessary in the setting of duodenal disruption. Laparoscopy in the treatment of traumatic duodenal perforation was first reported in 2010, and Tytgat et al described the approach in a 10-year-old boy with perforation over second part of duodenum. ${ }^{8,9}$ Donovan AJ et al (1966) shared his experience about 29 patients with traumatic perforation of the duodenum in which he stated the mortality of 14 percent. The author stated complications like duodenal fistula, secondary hemorrhage, pancreatic fistula and sepsis in such patients and emphasized on the importance of surgical intervention like primary repair to complex procedures like pancreatoduodenectomy with regard to the grade of injury. This is one of the oldest literatures in duodenal injury secondary to trauma. ${ }^{10}$

Rouse T et al (1984) published a case series in which one case was similar to our patient's presentation. The patient was a 21 years woman who suffered a road traffic accident who eventually underwent laparotomy. The patient had a $0.5 \mathrm{~cm}$ perforation on the anterior wall of the fourth part of the duodenum. The perforation was repaired in two layers. ${ }^{11}$ Considering more than 50 years (1966-2020) of literature, this case report is just a small example of how duodenal injuries can be undiagnosed and the significance of clinical suspicion in the patients presenting with blunt abdominal trauma should be understood. Road traffic accidents are one of the major causes of patients presenting to the Emergency Department. The type of treatment should be individualized and depends on the timing, location and extent of injury and the hemodynamic status of the patient. Open surgery is still the gold standard for patients that need surgical intervention. However, minimally invasive treatments are emerging as alternative methods.

The reason for this case being reported is firstly due to its infrequent occurrence. Isolated duodenal injuries are rare in patients presenting with blunt abdominal trauma. The presentation of the patient is similar to the literature. The timely diagnosis and intervention in this patient was crucial in preventing possible morbidity and mortality of the patient.

\section{REFERENCES}

1. Yao DC, Jeffrey Jr RB, Mirvis SE, Weekes A, Federle MP, Kim C, et al. Using contrast-enhanced helical $C T$ to visualize arterial extravasation after blunt abdominal trauma: incidence and organ distribution. American Journal of Roentgenology. 2002;178(1):17-20.

2. Celik A, Altinli E, Koksal N, Onur E, Sumer A, Uzun MA, et al. Management of isolated duodenal rupture due to blunt abdominal trauma: case series and literature review. Eur J Trauma Emerg Surg. 2010;36(6):573-8.

3. Omondi M, Mutua I, Kiptoon D. Isolated duodenal perforation at D4 following blunt abdominal trauma. Int J Surg Case Rep. 2020;72:596-8.
4. Ballard RB, Badellino MM, Eynon CA, Spott MA, Staz CF, Buckman RF. Blunt duodenal rupture: a 6-year statewide experience. Journal of Trauma and Acute Care Surgery. 1997;43(2):229-33.

5. Frick EJ, Pasquale MD, Cipolle MD. Small-bowel and mesentery injuries in blunt trauma. Journal of Trauma and Acute Care Surgery. 1999;46(5):920-6.

6. Luther A, Mann C, Hart C, Khalil K. Duodenal rupture secondary to blunt trauma from a football. Journal of surgical case reports. 2013;2013(1).

7. Ansari D, Toren W, Lindberg S, Pyrhonen HS, Andersson R. Diagnosis and management of duodenal perforations: a narrative review. Scand J Gastroenterol. 2019;54(8):939-44.

8. Huang CL, Lee JY, Chang YT. Early laparoscopic repair for blunt duodenal perforation in an adolescent. J Pediatr Surg. 2012;47(5):E11-4.

9. Tytgat S, Zwaveling S, Kramer W, van der Zee D. Laparoscopic treatment of gastric and duodenal perforation in children after blunt abdominal trauma. Injury. 2012;43(9):1442-4.

10. Donovan AJ, Hagen WE. Traumatic Perforation of the Duodenum. American Journal of Surgery. 1966;111:341-50.

11. Rouse T, Collin J, Daar A. Isolated injury to the intestine from blunt abdominal injury. British Journal of Accident Surgery. 1984;16:131-3. 